\title{
PLANETARY BOUNDARY LAYER HEIGHT AND WIND FIELD CHARACTERIZATION BY MEANS OF A LIDAR AT THE TEIDE OBSERVATORY IN THE CANARY ISLANDS
}

\author{
Michaël Sicard ${ }^{1,2}$, Sergio Tomás ${ }^{1}$, Adolfo Comerón ${ }^{1}$, Francisco Rocadenbosch ${ }^{1,2}$, Alejandro Rodríguez ${ }^{1}$, \\ Constantino Muñoz ${ }^{1}$, Oscar Batet ${ }^{1}$ \\ ${ }^{1}$ Remote Sensing Lab. (RSLAB), Department of Signal Theory and Communications, \\ Universitat Politècnica de Catalunya, C/ Jordi Girona, 1-3, Edif. D4-010, 08034 Barcelona, Spain \\ Email:msicard@tsc.upc.edu \\ ${ }^{2}$ Institut d'Estudis Espacials de Catalunya - Centre de Recerca de l'Aeronàutica i de l'Espai / Universitat \\ Politènica de Catalunya
}

\begin{abstract}
A lidar field campaign was performed between $30^{\text {th }}$ June and $4^{\text {th }}$ July 2007 in the Teide Observatory in the Canarian island of Tenerife to characterize the atmosphere of this astrophysical observation site in terms of nocturnal boundary layer height and wind fields. The nocturnal boundary layer height was found lower than $810 \mathrm{~m}$ in all cases and the aerosol optical thickness lower than 0.005 and 0.03 at 1064 and $532 \mathrm{~nm}$, respectively. Wind fields could hardly be retrieved because of faint signals and very weak wind velocities observed during the campaign.
\end{abstract}

Index Terms - boundary layer, wind field, lidar, site characterization

\section{INTRODUCTION}

The Teide Observatory (TO, 17'52'34' W; 28¹8'00' N) operated by the Instituto de Astrofísica de Canarias (IAC) is situated $2400 \mathrm{~m}$ above sea level in Izaña in the Canarian island of Tenerife. The observatory's geographical location, together with the clarity and excellent quality of the sky, makes it ideally suited for astronomy and solar studies. Standard site measurement equipment for the recording of essential site parameters (meteorological data, atmospheric turbulence characterization, etc.) is available and long records exist to establish reliable statistics of such parameters.

In that context, an intensive lidar field campaign has been performed in the $\mathrm{TO}$ between $30^{\text {th }}$ June and $4^{\text {th }}$ July 2007 in order to characterize the atmosphere in terms of nocturnal boundary layer (NBL) height and possibly of wind velocity profiles by tracking aerosol inhomogeneities. As a byproduct an estimate of the aerosol optical thickness (AOT) has also been obtained.

\section{LIDAR INSTRUMENT AND MEASUREMENT TYPES}

The lidar from the Universitat Politècnica de Catalunya is based on a frequency-doubled Nd:YAG laser delivering simultaneously pulses of approximately $160 \mathrm{~mJ}$ and $7 \mathrm{~ns}$ duration at 1064 and $532 \mathrm{~nm}$ [1]. The backscattered light is collected by an 8-inch diameter Schmidt-Cassegrain telescope and focused on one end of an optical-fiber bundle. At the other end of the bundle dichroic beamsplitters deflect the collected light towards 3 photodetectors. An avalanche photodiode-based receiver is used for the 1064- and the 532$\mathrm{nm}$ channels. A photomultiplier tube-based receiver is used at $607.4 \mathrm{~nm}$, corresponding to a Raman shift of the incident radiation at $532 \mathrm{~nm}$ produced by the atmospheric nitrogen. The system full overlap factor is reached at $400 \mathrm{~m}$.

The schedule of the 4 nights of measurements performed at the TO was from 2000 to 0800 UTC. Four types of measurements were performed as follows:

- Nocturnal cycle: static measurement of 30-min. duration at zenith with a 1-min. resolution. Product: Aerosol load and vertical aerosol distribution.

- Wind - vertical: repeated (60 times) static measurements of 4-s duration at zenith. Product: vertical component of the wind velocity.

- Wind - horizontal: repeated (60 times) alternative measurements between 2 lines of sight (LOS) separated by a $1-^{\circ}$ azimuth angle at an elevation angle of $10^{\circ}$. At each LOS the measurement is of 4-s duration. Product: horizontal components of the wind velocity at low heights.

- Wind - square: repeated (30 times) measurements along 4 LOS with a square shape (all LOS are separated by 1 or $2^{\circ}$ azimuth or elevation angle between each other). At each LOS the measurement is of $4 \mathrm{~s}$ duration. The starting elevation angle is $30^{\circ}$ or $45^{\circ}$. Product: horizontal components of wind velocity at higher altitudes than what the Wind - horizontal measurements allow. 


\section{NOCTURNAL BOUNDARY LAYER HEIGHT RETRIEVAL AND OPTICAL THICKNESS}

A total of 27 static measurements were performed over 4 nocturnal cycles. The AOT was measured by integrating between $400 \mathrm{~m}$ and $5000 \mathrm{~m}$ the extinction coefficient profiles retrieved by inverting the range square corrected signals (RSCS) with the Klett method and a constant lidar ratio of 30 sr. The aerosol load and vertical distribution are shown in Fig. 1 in a chronological manner. During the whole period a situation that can possibly be considered typical (although the measurement campaign was too short to gather statistically significant information as far as lidar-derived data are concerned) was met. The measurements show in general a low NPB layer with a height oscillating between 375 and $810 \mathrm{~m}$ and with a slight tendency to rise as the night goes by. Above the NBL, an optically thin aerosol distribution extends to typically a $1.5-\mathrm{km}$ height, generally monotonically decreasing, and in any case not higher than 2 $\mathrm{km}$. The variation of the aerosol concentration is much less visible at $532 \mathrm{~nm}$ because the signal is on the order of magnitude of the Rayleigh signal. In those situations the AOT between 400 and $5000 \mathrm{~m}$ is very low: typically less than 0.005 for the 1064-nm channel and less than 0.03 for the 532$\mathrm{nm}$ channel. If one extrapolates the average value found for the extinction at $400 \mathrm{~m}$ down to the ground the AOT usually increases around $50 \%$.

\section{WIND FIELDS}

The wind velocity determination by tracking aerosols' inhomogeneities is a method based in a correlation procedure. First, the fluctuations of the RSCS, a magnitude related to the aerosol concentration fluctuations, are calculated [2]. Then the calculations of the spatio-temporal cross correlation functions (CCF) between two pre-selected regions in space and time from both LOS is performed [3]. The iso-correlation curves of each CCF form an ellipse [4]. The displacement of the temporal coordinate of the CCF peak (the center of the ellipse) respect to the origin is the time shift along the transverse direction and the inclination of the ellipse in the auto-correlation function gives an estimation of the radial velocity.

Table 1 summarizes how well the wind components could be retrieved in all 3 measurement types. The "wind vertical" patterns were short-termed and temporally highly resolved to be able to detect aerosol motions within the NBL. In most cases no inhomogeneities were present or, when they were present, the weak aerosol content gave too weak lidar signalto-noise ratios (SNR) to be processed. Vertical wind profiles could be retrieved in $35 \%$ of the cases when sunrise was approaching and the AOT had generally increased. The profiles showed an almostzero vertical wind that could be observed in the fluctuation time series which were neither ascending nor descending. The highest and most reliable speeds, around $-0.34 \mathrm{~m} \cdot \mathrm{s}^{-1}$, were obtained between 500 and $800 \mathrm{~m}$ at the transition between the NBL and the free troposphere, as seen in the example shown in Fig. 2.

\begin{tabular}{|l|l|l|l|}
\hline & Wind - ver. & Wind - hor. & $\begin{array}{l}\text { Wind } \\
\text { squ. }\end{array}$ \\
\hline Total \# of meas. & 23 & 29 & 19 \\
\hline $\begin{array}{l}\text { Aerosols - } \\
\text { Wind meas. }\end{array}$ & $8(35 \%)$ & $3(10 \%)$ & $1(5 \%)$ \\
\hline $\begin{array}{l}\text { Aerosols - } \\
\text { No wind meas. }\end{array}$ & $9(39 \%)$ & $4(14 \%)$ & $8(42 \%)$ \\
\hline $\begin{array}{l}\text { No aerosols }- \\
\text { No wind meas. }\end{array}$ & $6(26 \%)$ & $22(76 \%)$ & $10(53 \%)$ \\
\hline
\end{tabular}

Table 1. Status of the wind measurements.

Only $8.5 \%$ of all horizontal wind measurements (Wind horizontal and W ind - square) allowed to retrieve horizontal wind indeed. The bad achievement of those measurements is due to the same reasons as for the vertical wind measurements (no inhomogeneities and poor lidar SNR) and also to the lack of contrast between the aerosol and the molecular signals that made difficult the distinction between both components. When horizontal wind was detected, reasonable wind speeds were detected until $300 \mathrm{~m}$ for the Wind - horizontal patterns, and until $600 \mathrm{~m}$ for the Wind square patterns. An example of Wind-horizontal measurement is shown in Fig. 3. The radial and the transversal wind velocities have been estimated in a range comprised between 20 and $160 \mathrm{~m}$ above ground level. Above $160 \mathrm{~m}$, which corresponds almost to $1 \mathrm{~km}$ in range inside the NBL, the profiles are of very bad quality. The transversal wind speed is measured using the entire temporal window and for the radial wind speed only a specific window centered temporally around the main inhomogeneity (see arrow in Fig. 3a and 3b) is considered. Two main regions can be observed: $[50 ; 70 \mathrm{~m}$ ] in which the transversal and radial speed are on the order of -2 and $+0.75 \mathrm{~m} \cdot \mathrm{s}^{-1}$, respectively, and $[80 ; 120 \mathrm{~m}]$ in which the transversal and radial speed are on the order of -4 and $-1 \mathrm{~m} \cdot \mathrm{s}^{-1}$, respectively. The resulting mean wind velocity and direction (W and SW-W) are in agreement with previous studies [5]. In all the other cases of the campaign (when wind speed could be retrieved), the speeds found are lower than in the examples shown here.

\section{CONCLUSIONS}

A lidar filed campaign was carried out in the Teide Observatory between $30^{\text {th }}$ June and $4^{\text {th }}$ July 2007. Low NBL heights were observed $(<810 \mathrm{~m})$ and no aerosols were detected higher than $2 \mathrm{~km}$. Very small AOTs were measured: 0.005 and 0.03 at 1064 and $532 \mathrm{~nm}$, respectively. The vertical motion of the aerosols was null nearly during the whole campaign. Horizontal wind speeds were detected in very few cases (in $8.5 \%$ of the measurements) due to the lack of 

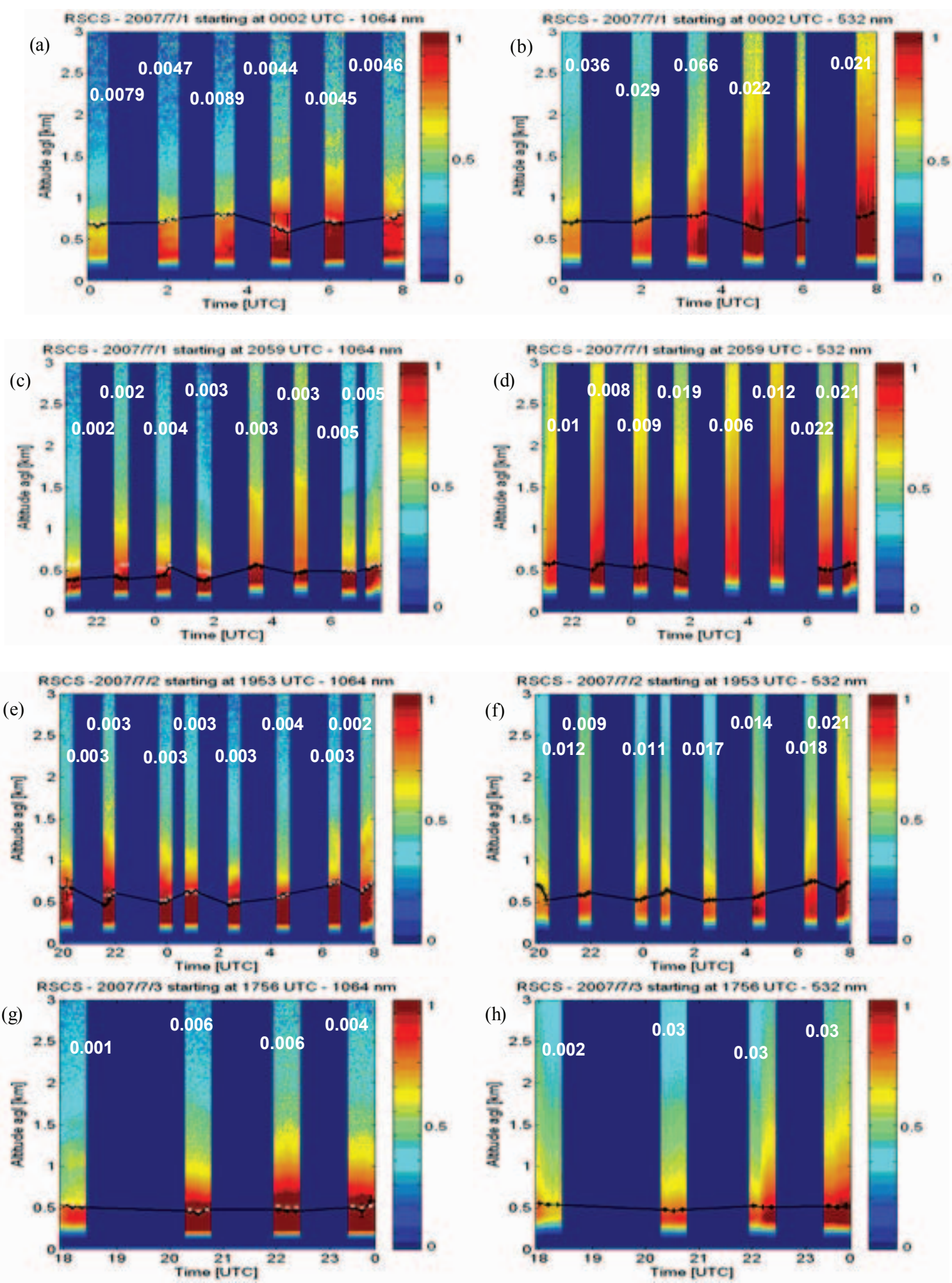

Fig. 1. RSCS at $1064 \mathrm{~nm}$ (left) and $532 \mathrm{~nm}$ (right) on the night of $30^{\text {th }}$ June (a, b), $1^{\text {st }}$ July (c, d), $2^{\text {nd }}$ July (e, f) and $3^{\text {rd }}$ July (g, h). The NBL height measured with a 10-min. resolution is represented by black plusses. On the left figures, the NBL height from the 532-nm channel is reproduced by white circles. The white numbers indicate the AOT calculated for each 30 -min. integrated profile. 


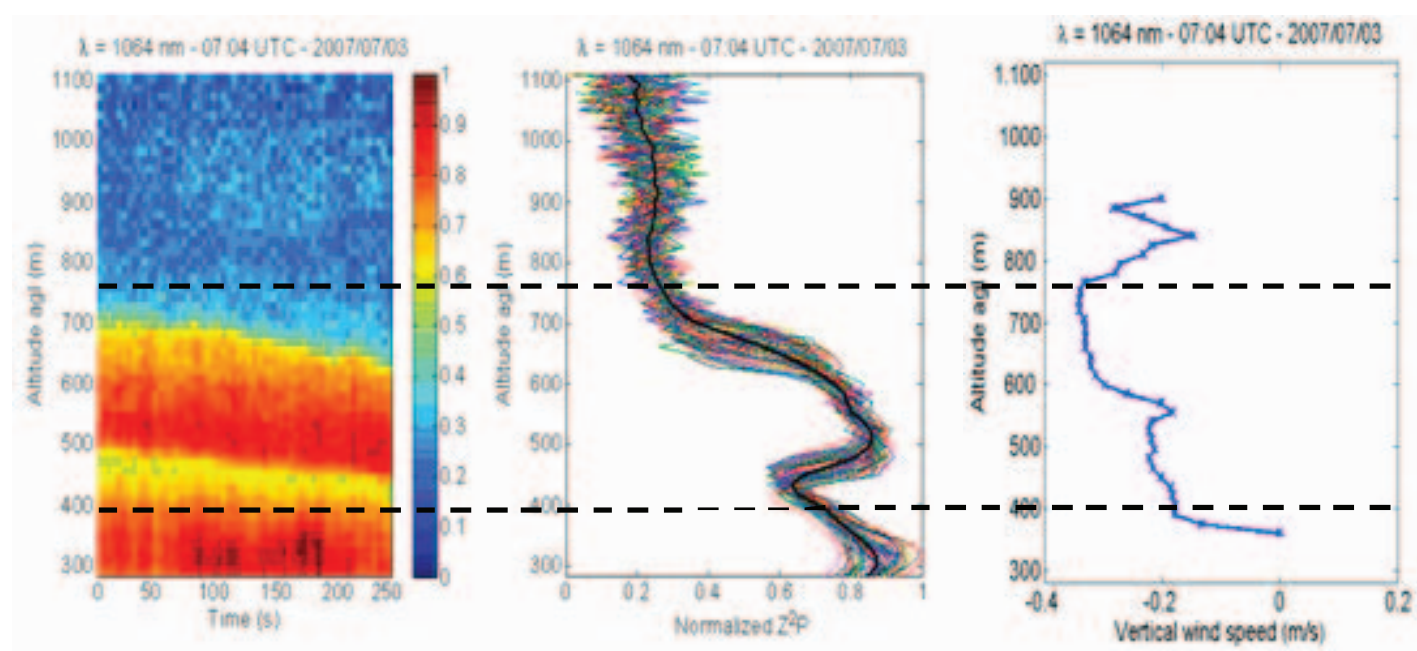

Fig. 2. Example of a Wind - vertical measurement: 4-min.t ime series (left) of the RSCS at $1064 \mathrm{~nm}$. The 60 corresponding profiles are represented in the center figure. The black curve shows the mean profile. The vertical wind speed is shown in the right figure.

(a)

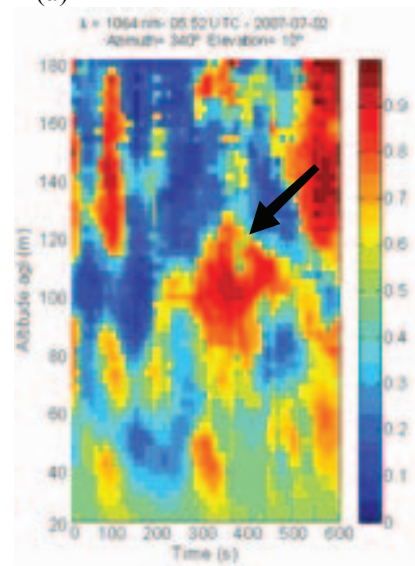

(b)

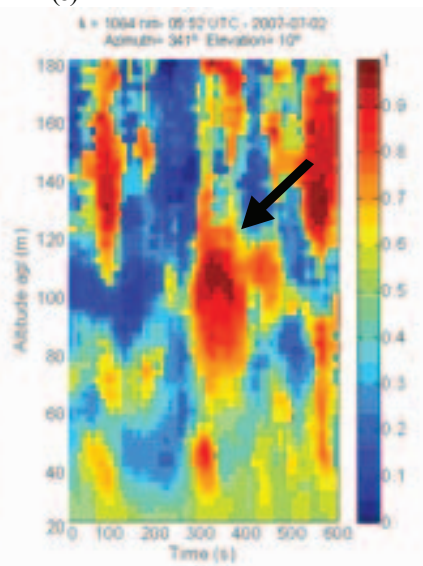

(c)

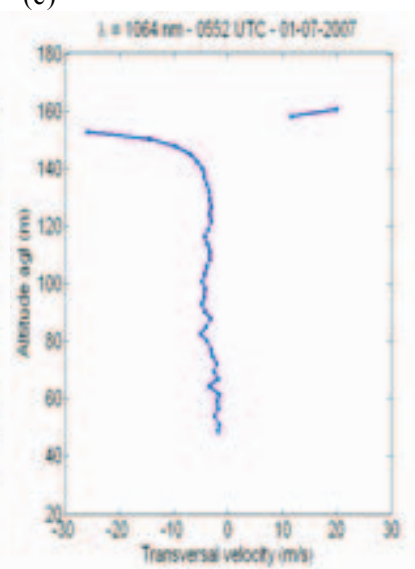

(c)

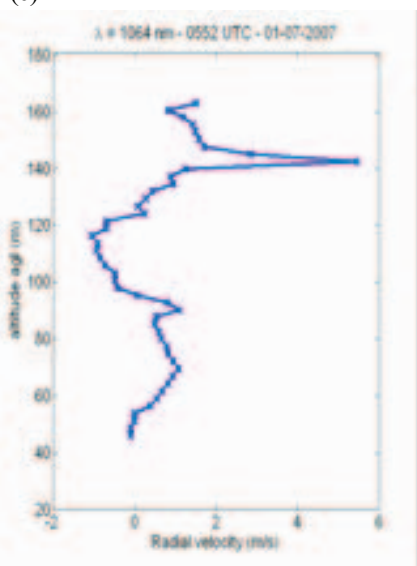

Fig. 3. Example of a Wind - horizontal measurement: fluctuations in RSCS at 1064 nm along 2 LOS: $340^{\circ}$ (a) and $341^{\circ}$ (b). The transversal and the radial wind speeds are shown in (c) and (d), respectively.

inhomogeneities or/and poor lidar SNR. When horizontal wind speeds could be retrieved, speeds not higher than 2 $\mathrm{m} \cdot \mathrm{s}^{-1}$ were measured.

\section{Acknowledgments}

This work was supported by the EU and FEDER funds under the EARLINET-ASOS project (EU Coordination Action, contract $\mathrm{n}^{\mathrm{o}} 025991 \quad$ (RICA)) and the "Technology development programme towards a European Extremely Large Telescope" project (EU Specific Support Action, contract $\mathrm{n}^{\circ} 011863$ (RIDS)); by the ESA under the project 21487/08/NL/HE; and by the MEC (Spanish Ministry of Education and Science) and FEDER funds under the project TEC2006-07850/TCM, and the Complementary Actions CGL20007-28871-E/CLI and CGL2006-26149-E/CLI. M. Sicard thanks the MEC for his Ramón y Cajal position.

\section{REFERENCES}

[1] Rocadenbosch F., M. Sicard, A. Comerón, J.M. Baldasano, A.
Rodríguez, R. Agishev, C. Muñoz, M.A. López, D. GarcíaVizcaino, "The UPC scanning Raman lidar: an engineering overview", Proc. of the 21 ILt ILRC, Vol. 1, pp. 69-70, 2002.

[2] S. Tomás, M. Sicard, J. Masjuan, M. N. Md. Reba, C. Muñoz, F. Rocadenbosch, "A wind speed and fluctuation simulator for characterizing the wind lidar correlation method". Proc. of the IGARSS'07, ISBN: 1-4244-1212-9, pp.2779 - 2782, 2007.

[3] W. T. Buttler, C. Soriano, J M. Baldasano, G. H. Nickel, "Remote sensing of three-dimensional winds with elastic lidar: explanation of maximum cross-correlation method", BoundaryLayer Meteorol., 97, 305-328, 2001.

[4] G. J., Kunz, "Wind Measurements with an Incoherent Lidar", TNO Physics and Electronics Laboratory, FEL-93-A040, August 1993.

[5] A. M. Varela, C. Muñoz-Tuñón, A. G. De Gurtubai, C. Saviron, "Site-testing results at the Teide Observatory", ASP Conference Series, Vol. 266, pp. 454-464, 2002. 